Methods: Cases were identified based on their recorded contacts with community mental health teams between July 2003 and June 2005. The observed incidence was compared with recently published treated incidence rates through indirect standardization. The sensitivity of the EP flags for identifying cases was calculated. Service utilizati on, as measured by treatment days per quarter, was compared between EP clients and other groups.

Results: There were 2475 cases identified in 20042005 , which is $40 \%$ higher than expected. The EP flags failed to identify these cases (sensitivity 7\%-39\%). Psychosis clients had a significantly higher level of service utilization: clients with a prior psychosis diagnosis had the highest mean treatment days (7.7), followed by firstepisode psychosis (6.5), bipolar/mania (5.7) and other diagnoses (3.5). These differences persisted after controlling for age and AHS $(P<0.001$ for all contrasts).

Conclusions: The incidence of first-episode psychosis was higher than expected possibly because of inconsistencies in diagnostic recording in 2003-2004. The EP flags are underused and fail to identify clients with first-episode psychosis, whose service utilizations differs from other clients.

\section{A dissociation of structure and function in the auditory cortex of patients with schizophrenia}

\section{Gavrilescu', S Rossell', R Maitra², D Copolov³, T Shea', K Henshall ${ }^{4}$, A Sergejew ${ }^{5}$, G Egan $^{6}$}

${ }^{1}$ Mental Health Research Institute; ${ }^{2}$ National Brain Research Centre, India; ${ }^{3}$ Monash University, Melbourne, Australia; ${ }^{4}$ The University of Melbourne, Melbourne, Australia; ${ }^{5}$ Maroondah Hospital; and ${ }^{6}$ Howard Florey Institute, Melbourne, Australia

Introduction: A number of investigative methods, for example, structural, metabolic and functional, have indicated a role for the auditory cortices in the pathophysiology of schizophrenia. However, few studies have completed multimodal investigations in the same participants. The aim of this study was to explore the structure and function of the auditory cortices in patients with schizophrenia.

Methods: Structural and functional magnetic resonance imaging (fMRI)images were acquired for 27 patients with schizophrenia and 16 normal controls. Heschl's gyrus (HG) and planum temporale (PT) were manually delineated on the structural scans of all subjects in both hemispheres. A structural laterality coefficient was calculated based on region of interest (ROI) volumes. The fMRI data were recorded while the subjects passively listened to semantically neutral words. The functional data were then coregistered with the structural images, and a functional laterality coefficient was calculated based on the number of activated voxels in the ROIs. The structural and functional laterality coefficients were compared across the groups using ANOVA.

Results: For HG, patients showed significantly reduced structural leftward laterality and increased functional rightward laterality in contrast to the control group. For PT, we found no structural differences between the groups, all groups were symmetric, while functionally again we found increased rightward laterality for the patients when compared with controls.

Conclusions: Patients with schizophrenia showed both reduced volume and poor activation of their left hemisphere auditory cortices. It therefore appears to be the case that language functions normally processed in the left hemisphere are processed in the right hemisphere in these individuals.

\section{Reduced interhemispheric connectivity in the central auditory system of patients with auditory hallucinations}

\section{Gavrilescu', S Rossell', G Stuart', T Shea', K Henshall' ${ }^{2}$ A Sergejew ${ }^{3}$, D Copolov', G Egan ${ }^{5}$}

${ }^{1}$ Mental Health Research Institute; ${ }^{2}$ The University of Melbourne, Melbourne, Australia; ${ }^{3}$ Maroondah Hospital; ${ }^{4}$ Monash University, Melbourne, Australia; and ${ }^{5}$ Howard Florey Institute, Melbourne, Australia

Introduction: Evidence from neuroanatomical, neurophysiological and functional neuroimaging studies has indicated that patients prone to auditory hallucinations (AHs) have structural and functional abnormalities affecting brain regions involved in a number of cognitive and emotional functions. Our previous work has shown deficits in the central auditory system of patients with AHs. In the current study, we hypothesized that this deficit is the result of interhemispheric pathway dysfunctions of the primary and secondary auditory cortices (PAC and SAC). The aim of this study was to investigate the interhemispheric functional connectivity (FC) of PAC and SAC in patients with AHs using functional magnetic resonance imaging (fMRI) data. Methods: Fourteen patients with schizophrenia with AHs, 13 patients with schizophrenia without AHs (nonAHs) and 16 normal controls were scanned while passively listening to semantically neutral words and at rest. Regions of interest were defined for each subject over PAC and SAC in both hemispheres. For the stimulus data, the stimulus effects were identified and regressed out of these time courses to estimate a 'rest-like' FC.

Results: The interhemispheric FC was significantly reduced for the AHs group both for stimulus and resting-state data, while the non-AHs and controls had similar connectivity values. 
Conclusions: Interhemispheric connectivity between the auditory cortices permits the association of basic auditory information with higher order language processing as the left and right auditory cortices are primarily responsible for different linguistic functions. Without efficient integration of auditory and linguistic experiences, errors in the interpretation of auditory phenomenon may occur; thus, this failure to integrate may result in the experience of AHs.

\section{Responding to deliberate self- harm among school students: the development and evaluation of evidence-based training}

\section{S Gook, J Robinson, A Yung, P McGorry, HP Yuen, T Jorm}

ORYGEN Research Centre, Melbourne, Australia

Background: Deliberate self-harm is prevalent among school students; however, evidence-based training on responding to this is limited.

Aims: The aims of the study were to develop and evaluate a training package for school staff to assist them manage self-harm among young people.

Methods: The training was a 2-day evidence-based package specifically designed for school staff. It aimed to better enable participants to understand and recognize self-harm and mental illness; improve the levels of confidence and perceived skill of participants in their ability to identify and manage self-harm, mental illness and risk and to improve attitudes of participants toward young people engaging in self-harm. The evaluation adopted a pretest/posttest design. Baseline measures were administered immediately prior to the training and were repeated immediately after the training. They will also be repeated at 6-month follow-up.

Results/Conclusions: The training was delivered to 213 school welfare staff across Victoria between May and August. Part 1 of the evaluation is now complete and analyses are under way. The findings will be presented and the overall efficacy of the training will be discussed.

\section{Increasing clinical monitoring of metabolic health in patients treated with antipsychotic medication}

\section{Graydon ${ }^{1,2}$, J Kulkarni', A deCastella ${ }^{1}$}

'Alfred Psychiatry Research Centre, The Alfred and Monash University, School of Psychology, Psychiatry \& Psychological Medicine, Melbourne, Australia; and ${ }^{2}$ Murdoch University, Perth, Australia
A priority in the treatment of schizophrenia and related conditions is to bring symptoms such as psychosis under control. To date, the most effective treatment modality to achieve this goal is pharmacotherapy. Unfortunately, a common side-effect of antipsychotic medication is weight gain, which in turn is linked to other serious physical conditions such as diabetes. The metabolic side-effects of taking antipsychotics can so impact upon the patient that they choose to skip or discontinue taking medication, which puts them at increased risk of relapse. Thus, improving the general health of people with schizophrenia and controlling the side-effects of antipsychotic medication are important clinical goals. The monitoring of patients' weight, body mass index, lipids and blood pressure is an important component of maintaining physical health and is an important factor in maintaining adherence to medication regimes. If metabolic side-effects do develop, use of a monitoring instrument would alert the clinician to the fact. We present an overview of the issues around monitoring of metabolic sideeffects, as well as an overview of a study currently being conducted, which assesses clinician's monitoring practices. The primary aim of this study was to assess metabolic monitoring practices by clinicians with a range of experience. A set of metabolic monitoring guidelines will be implemented, and the same clinicians will be reassessed over a 6-month period. This research will result in more consistent monitoring of metabolic side-effects, leading to improved mental and physical health outcomes for patients receiving antipsychotic medications.

\section{Perceived burden in carers of patients with mild Alzheimer's disease: the effect of patient insight}

\author{
K Greenop, 0 Almeida, K Cox, L Flicker, J Foster, \\ S Starkstein, N Lautenschlager
}

University of Western Australia, Perth, Australia

Background: Caring for a family member with dementia can cause stress; possible causes are the patient's lack of insight, poor functional ability or poor health. This study examined the association between level of insight in the patient and perceived burden in the carer.

Methods: Twenty-four patients with mild Alzheimer's disease and their carers were recruited from Perth Metropolitan Memory clinics and community advertising. Patients were administered a neuropsychological battery and completed the Geriatric Depression Scale (GDS), Dysexecutive (DEX) questionnaire and SF-36 health survey. Carers were interviewed separately on 